\title{
Treatment Outcomes of Patients With Cervical Intraepithelial Neoplasia or Invasive Carcinoma Who Underwent Loop Electrosurgical Excision Procedure
}

\author{
Jakkapan Khunnarong $^{\mathrm{a}, \mathrm{d}}\left(\mathbb{0}\right.$, Nitinan Bunyasontikul $^{\mathrm{a}, \mathrm{b}}\left(\mathbb{0}\right.$, , Siriwan Tangjitgamol $^{\mathrm{a}, \mathrm{c}}(\mathbb{C})$
}

\begin{abstract}
Background: This study aimed to evaluate the treatment outcomes of cervical intraepithelial neoplasia (CIN) or cancer patients who underwent loop electrosurgical excision procedure (LEEP) in terms of primary outcome and factors associated with persistence/recurrence.

Methods: Patients with CIN or cancer who underwent LEEP from January 2007 to December 2015 were reviewed. Data collected were age, parity, menopausal status, human immunodeficiency virus (HIV) infection, smoking, cervical cytology, histopathology from cervical biopsy and LEEP including margin status, final histopathology, and follow-up data.
\end{abstract}

Results: The mean age of 385 patients was $41.9 \pm 10.8$ years (range 18 - 79 years). Majority were multiparous (81.6\%) and premenopausal (78.2\%). There were $15.3 \%$ of patients with HIV infection. The most common cervical cytology was high-grade squamous cell intraepithelial lesion (HSIL, 44.1\%), followed by atypical squamous cells of undetermined significance (ACS-US, 21\%). Minor complications of bleeding or infection from LEEP were encountered in $7.3 \%$. Among 153 patients (39.7\%) who had positive margin(s), 43 underwent second LEEP, whereas 76 had hysterectomy. From all patients, 47 had failure after treatment $(12.2 \%)$, being either persistence $(30$ patients; $7.8 \%$ ) or recurrence (17 patients; $4.4 \%$ ). Factors associated with persistence or recurrence by multivariate analysis were age $\geq 55$ years old, HIV infection, final diagnosis of invasive cancer, and positive endocervical margin or both ecto- and endo- cervical margins.

Conclusions: LEEP had low rate of persistence/recurrence. Age $\geq$ 55 years old, HIV infection, final diagnosis of cancer, and positive

Manuscript submitted May 15, 2021, accepted June 15, 2021

Published online July 10, 2021

aDepartment of Obstetrics and Gynecology, Faculty of Medicine, Vajira Hospital, Navamindradhiraj University, Bangkok, Thailand

bObstetrics and Gynecology Section, Somdejprapinklao Hospital, Bangkok, Thailand

'Women's Health Center, MedPark Hospital, Bangkok, Thailand

${ }^{\mathrm{d}}$ Corresponding Author: Jakkapan Khunnarong, Faculty of Medicine, Vajira Hospital, Navamindradhiraj University, 681 Samsen Road, Dusit, Bangkok 10300, Thailand. Email: jak020@hotmail.com

doi: https://doi.org/10.14740/wjon1391 endocervical or both endo- and ecto- surgical margin(s) were significantly associated with persistent or recurrent diseases.

Keywords: CIN; Microinvasive carcinoma; LEEP; Persistence; Recurrence

\section{Introduction}

Cervical cancer is the fourth most common cancer in women after breast, colorectal and lung cancer worldwide. The estimated new cases and deaths in 2018 were 569,847 and 311,365 , respectively [1]. The incidence and mortality were higher in low and medium human development index (HDI) countries compared to lower mortality in high HDI regions. In Thailand 2018, cervical cancer is the second common cancer after breast cancer with 8,622 new cases and 5,015 deaths [1].

One effective means to reduce cervical cancer incidence and death is a detection and treatment of precancerous lesions of cervix or cervical intraepithelial neoplasia (CIN). The CIN, especially high grade (CIN 2/3), is a lesion that can progress to invasive cancer if left untreated [2].

Cervical conization is an excisional procedure for diagnosis or treatment of CIN. The procedure allows thorough histologic examination of the cervix, whereas fertility function is preserved. The original technique of conization which had long been used is cold knife conization (CKC). However, this $\mathrm{CKC}$ requires an experienced or well-trained gynecologist, general anesthesia, and hospitalization. Subsequent conization technique using an electrical-transmitted wire loop instead of a knife, so-called a loop electrosurgical excision procedure (LEEP), was initially developed by Cartier et al in 1981 [3] and is commonly used worldwide in the current era.

LEEP has been proven to be equivalent to $\mathrm{CKC}$ in terms of the indications and treatment outcomes including complications and cure rate if performed properly [4]. Previous studies showed $73 \%$ to $99 \%$ cure rate of CIN by LEEP $[5,6]$. The wide range of cure rate or persistent or recurrent disease in each study could be influenced by various risk factors, such as, parity and age of patients, immune status especially human immunodeficiency virus (HIV) infection, status of resected margins, etc.

Our institution used LEEP for diagnosis and/or treatment 
of CIN and other cervical lesions for many years. This study aimed to evaluate the treatment outcomes after LEEP including complications and rates of persistence or recurrence. Factors associated with persistence/recurrence were also studied.

\section{Materials and Methods}

The study was approved by the Ethics Committee of the institution. This study was conducted in compliance with the ethical standards of our institution on human subjects as well as with the Helsinki Declaration. Inclusion criteria were patients who were treated by LEEP for CIN or microinvasive cervical cancer from January 2007 to December 2015 and had followup visits in our institution. The patients who had incomplete medical record were excluded.

As a general practice in our institution, patients with abnormal cervical cytology would undergo colposcopic examination. The examination was performed under green filter, and normal lighting after application of $5 \%$ acetic acid to visualize and map cervical lesions. Biopsy with or without endocervical curettage (ECC) was carried out as appropriate. LEEP would be consequently performed for unsatisfactory colposcopy or biopsy results of high-grade squamous cell intraepithelial lesions (HSILs) defined as CIN 2-3 or microinvasive carcinoma (MIC). A loop electrode diameter of $10-25 \mathrm{~mm}$ setting with a cut or blend modes (mixed cut and coagulation) and a power of $40-50 \mathrm{~W}$ was used. Hemostasis was achieved with electric ball coagulation or application of Monsel's solution. The procedure was generally performed by a gynecologic oncologist or a gynecologic oncology fellow in training under supervision.

The formalin-fixed LEEP specimen was grossly examined before serial tissue sectioning. Histologic examination included histopathology, size, and depth of lesion as well as margin status. Positive margin was detailed as ecto- (ectomargin), endo-cervical margins (endomargin) or both ecto- and endomargins. Presence or absence of dysplastic epithelium in the curettage specimen was also reported. The patients who had positive margin(s) were counseled for options of follow-up, reLEEP or hysterectomy at the discretion of the physician based on the women history of parity, severity of lesion, coincidental gynecologic pathology, and the women's preference.

Except for patients with positive surgical margin(s) who would have the first follow-up at 3 months after LEEP, the others were evaluated by pelvic examination and cervical cytology every 6 months after the procedure for a period of 2 years, then yearly afterwards. Colposcopy or cervical biopsy was performed as indicated.

Data collected from medical charts and electronic database were age, underlying disease, parity, smoking status, cervical cytology result, pathologic results (cervical biopsy, LEEP and its margin status, and hysterectomy specimens), period of follow-up, and events of persistence or recurrence. Persistence was diagnosed if subsequent CIN was evidenced within 6 months after LEEP, and recurrence if CIN was found after 6 months $[7,8]$.

Demographic data, types of abnormal cervical cytology and histology were analyzed by descriptive statistics. Number with percentage and mean with standard deviation (SD) or median with range were used to describe categorical and continuous variables, respectively. Chi-square or Fischer exact test was used to test the relationships between clinic-pathologic features and events of persistence or recurrence. Progressionfree survival was analyzed by Kaplan-Meier and risk factors were compared with Log-rank test. P values equal or less than 0.05 were considered statistically significant. All data were analyzed using IBM SPSS Statistics for Windows, Version 22.0 (IBM Corp., Armonk, NY).

\section{Results}

During the study period, 401 patients underwent LEEP in our institution. Fifteen patients were excluded: 11 with final diagnosis of benign tissue (negative for malignancy or cervicitis) and five who were lost to follow-up before 6 months after LEEP. The mean age of 385 patients included in the study was $41.9 \pm 10.8$ years (median 41.5 years, range $18-79$ years). Almost half of patients had parity of 2 or 3 (median parity of 2 , range $0-6)$. Majority were pre-menopause $(78.2 \%)$. Nearly all patients denied history of smoking and $15.3 \%$ (59 patients) had HIV infection.

Except four patients who had normal cervical cytology but underwent colposcopic examination because of chronic leukorrhea or abnormal cervical findings from pelvic examination, the other 381 patients had abnormal cervical cytology. Squamous cell abnormality (367 cases or 95.3\%) was more common than glandular cell type. Almost half were high grade squamous intraepithelial lesions (HSIL, 44.1\%), followed by atypical squamous cells of undetermined significant (ASCUS, $21.0 \%$ ) and low-grade squamous intraepithelial lesions (LSILs, 18.7\%). Atypical glandular cells (AGCs) were presented in 3.4\% and adenocarcinoma in situ (AIS) in only one.

From 385 patients, seven were treated by LEEP following colposcopy without cervical biopsy. The most common histopathology among 378 patients who underwent colposcopic directed biopsies were CIN 2/3 (76.9\%), followed by CIN 1 (9.4\%) and carcinoma in situ (5.5\%). Demographic data of the patients, cervical cytology and histology of biopsy are presented in Table 1.

Only minor post-operative complications of bleeding (16 patients, $4.2 \%$ ) or local infection (12 patients or 3.1\%) were reported. The histopathology from LEEP specimens were CIN $2 / 3$ in 321 patients $(83.4 \%)$, CIS or AIS in $27(7.0 \%)$, and cervical cancer in $21(5.5 \%)$. Among patients with invasive cancer, 18 had squamous cell carcinoma (SCC; 15 of microinvasive or FIGO stage IA1 and IA2 and three of FIGO stage IB1), whereas three had adenocarcinoma of FIGO stage IB1). Positive margin was evidenced in 153 cases (39.7\%): positive endomargin in $79(20.5 \%)$, ectomargin in $40(10.4 \%)$, and both margins in $34(8.8 \%)$. The primary outcomes after LEEP are demonstrated in Table 2.

After primary LEEP, re-LEEP was performed in 36 out of 153 patients $(23.5 \%)$ with positive surgical margins. The patients with positive endocervix or positive both margins underwent re-LEEP significantly more frequent than those with 
Table 1. General Characteristics of Patients Underwent LEEP $(\mathrm{N}=385)$

\begin{tabular}{|c|c|}
\hline Characteristic & n (\%) \\
\hline Age (mean), years & $41.9 \pm 10.8$ \\
\hline \multicolumn{2}{|l|}{ Parity } \\
\hline Null & $71(18.4)$ \\
\hline 1 & $114(29.6)$ \\
\hline $2-3$ & $179(46.5)$ \\
\hline$\geq 4$ & $21(5.5)$ \\
\hline Pre-menopause & $301(78.2)$ \\
\hline Smoking & $6(1.6)$ \\
\hline HIV infection & $59(15.3)$ \\
\hline \multicolumn{2}{|l|}{ Initial cervical cytology } \\
\hline ASC-US & $81(21.0)$ \\
\hline ASC-H & $26(6.8)$ \\
\hline LSIL & $72(18.7)$ \\
\hline HSIL & $170(44.1)$ \\
\hline CIS & $3(0.8)$ \\
\hline $\mathrm{SCC}$ & $15(3.9)$ \\
\hline $\mathrm{AGC}$ & $13(3.4)$ \\
\hline AIS & $1(0.3)$ \\
\hline NILM & $4(1.0)$ \\
\hline \multicolumn{2}{|l|}{ Cervical biopsy results } \\
\hline Not done & $7(1.8)$ \\
\hline Negative for CIN or inflammation & $17(4.5)$ \\
\hline CIN 1 & $36(9.4)$ \\
\hline CIN 2/3 & $296(76.9)$ \\
\hline CIS & $21(5.5)$ \\
\hline SCC & $7(1.8)$ \\
\hline AIS & $1(0.3)$ \\
\hline
\end{tabular}

HIV: human immunodeficiency virus; ASC-US: atypical squamous cells of undetermined significance; ASC-H: atypical squamous cells cannot exclude HSIL; LSIL: low-grade squamous cell intraepithelial lesion; HSIL: high-grade squamous cell intraepithelial lesion; CIS: carcinoma in situ; SCC: squamous cell carcinoma; AGC: atypical glandular cells; AIS: adenocarcinoma in situ; NILM: negative for intraepithelial lesion or malignancy; $\mathrm{CIN}$ : cervical intraepithelial neoplasia.

positive ectocervix: $26.6 \%$ with positive endocervix (21 of 79 patients) and $29.4 \%$ with positive both margins (10 of 34 patients) compared to $12.5 \%$ (five of 40 patients), respectively $(\mathrm{P}<0.001)$. Positive margin was found from the subsequent LEEP in only four patients $(9.3 \%)$. Of note, seven patients with negative margin also had re-LEEP due to clinical suspicion of invasive cancer (pathology of CIN3 with close margin from the first LEEP) in two patients and for recurrence in the other five patients.

Overall, hysterectomies were subsequently performed in 76 patients of persistent or recurrent CIN (37 patients, $48.7 \%$ ), other gynecologic conditions (20 patients, 26.3\%) and micro-
Table 2. Histologic Outcome After First LEEP ( $\mathrm{N}=385)$

\begin{tabular}{ll}
\hline Results & n (\%) \\
\hline $\begin{array}{l}\text { Complications } \\
\text { Bleeding }\end{array}$ & $16(4.2)$ \\
Infection & $12(3.1)$ \\
Final diagnosis & \\
\hline CIN 1 & $16(4.2)$ \\
CIN 2/3 & $321(83.4)$ \\
CIS & $24(6.2)$ \\
Squamous cell carcinoma stage IA1 & $14(3.6)$ \\
Squamous cell carcinoma stage IA2 & $1(0.2)$ \\
Squamous cell carcinoma stage IB1 & $3(0.8)$ \\
AIS & $3(0.8)$ \\
Adenocarcinoma stage IB1 & $3(0.8)$ \\
Margin status after first LEEP & \\
Negative margin & $232(60.3)$ \\
Positive ectomargin & $40(10.4)$ \\
Positive endomargin & $79(20.5)$ \\
\hline Positive both margins & $34(8.8)$ \\
\hline
\end{tabular}

CIN: cervical intraepithelial neoplasia; CIS: carcinoma in situ; AIS: adenocarcinoma in situ; LEEP: loop electrosurgical excision procedure.

invasive or invasive carcinoma (19 patients, $25.0 \%$ ). To be noted, nine patients had re-LEEP before proceeding to hysterectomy due to persistent of CIN (five patients), having cancer or other gynecologic conditions (two patients each). Pathology from hysterectomy specimens confirmed residual CIN in 26 patients (34.2\%) and cancer in five (6.5\%). The other gynecologic diseases were also reported and found almost half had myoma uteri or adenomyosis with small number both benign and malignant ovarian tumors. The outcome and subsequent histology of LEEP and hysterectomy are shown in Table 3.

After the median follow-up time of 63.0 months (range 7.4 - 157.3 months), we found 47 patients (12.2\%) had failure after treatment: being persistent disease in 30 patients $(7.8 \%)$ and recurrent CIN in 17 (4.5\%). The median interval from LEEP to a diagnosis of persistence was 3.8 months (range $0.8-5.9$ months), whereas the median interval to recurrence was 13.1 months (range 7.1 - 83.2 months). We studied the association between pre- and post-operative features with failure after LEEP. Age $\geq 55$ years, HIV infection, initial cervical cytology of HSIL or higher results, final diagnosis of microinvasive or invasive carcinoma, and positive endo-cervical or positive both ecto- and endo-cervical margin of LEEP were significantly associated with persistence or recurrence by univariate analyses.

By multivariate analyses, we found old age, HIV infection, final diagnosis of cancer, and positive endo-margin or positive endocervical margin remained significant factors associated with failure after LEEP. The hazard ratios (HRs) for age of 55 or older or HIV infection were $4.8(95 \%$ confidence interval $(\mathrm{CI}): 1.5-15.0, \mathrm{P}=0.008)$ and $3.1(95 \% \mathrm{CI}: 1.3-7.1, \mathrm{P}=$ 
Table 3. Outcome of Subsequent LEEP or Hysterectomy

\begin{tabular}{|c|c|}
\hline Results & n (\%) \\
\hline \multicolumn{2}{|l|}{ Indications of subsequent LEEP $(n=43)$} \\
\hline Positive margins(s) & $38(88.4)$ \\
\hline Recurrent CIN & $5(11.6)$ \\
\hline \multicolumn{2}{|l|}{ Margin status after subsequent LEEP $(n=43)$} \\
\hline Negative margin & $39(90.7)$ \\
\hline Positive margin & $4(9.3)$ \\
\hline \multicolumn{2}{|c|}{ Pathology of subsequent LEEP specimens $(n=43)$} \\
\hline Negative or cervicitis & $14(32.6)$ \\
\hline CIN 1 & $11(25.6)$ \\
\hline CIN $2 / 3$ & $16(37.2)$ \\
\hline $\mathrm{SCC}$ & $2(4.6)$ \\
\hline \multicolumn{2}{|l|}{ Indications of hysterectomy $(\mathrm{n}=76)$} \\
\hline Persistent or recurrent CIN & $37(48.7)$ \\
\hline Microinvasive or invasive carcinoma & $19(25.0)$ \\
\hline Other gynecologic conditions & $20(26.3)$ \\
\hline \multicolumn{2}{|l|}{ Pathology of hysterectomy* $(n=76)$} \\
\hline Negative finding & $20(26.3)$ \\
\hline Residual CIN & $26(34.2)$ \\
\hline $\mathrm{MIC}$ & $3(3.9)$ \\
\hline Invasive SCC or adenocarcinoma & $2(2.6)$ \\
\hline Myoma or adenomyosis & $35(46.1)$ \\
\hline Benign ovarian tumor & $7(9.2)$ \\
\hline Epithelial ovarian cancer & $1(1.3)$ \\
\hline
\end{tabular}

*Some hysterectomy specimens had more than one pathology. LEEP: loop electrosurgical excision procedure; CIN: cervical intraepithelial neoplasia; SCC: squamous cell carcinoma; MIC: microinvasive carcinoma.
0.009), respectively. For final diagnosis, the patients with microinvasive or invasive cancer had HR 6.0 (95\% CI: 1.9 - 18.7, $\mathrm{P}=0.02$ ). On the other hand, HR for positive endo-cervical margin was $1.7(95 \% \mathrm{CI}: 1.2-2.7, \mathrm{P}=0.02)$ and increased to 10.1 (95\% CI: $4.2-24.2, \mathrm{P}<0.001)$ for positive both margins. The persistence/recurrence according to demographic data or pathologic results is shown in Table 4 and Figure 1.

\section{Discussion}

Treatment of CIN especially CIN 2-3 is crucial to prevent a progression of these pre-invasive to invasive cervical cancer $[9,10]$. Different excisional modalities of cervix by CKC, laser conization or LEEP are all acceptable as a diagnostic or therapeutic procedure. LEEP has become a more common technique used in a current clinical practice due to its comparable efficacy to treat CIN 2/3, persistent CIN 1 , and MIC, less complications and lower cost than CKC. Nevertheless, one major concern of this cervical excision (regardless of the method used) is the persistence or recurrence reflecting failure of treatment.

Various rates of cure or failure from each study may depend on many factors aside from the surgical procedure itself, e.g., personal features of the patients, practice of surgeon including the skill and aggressive surgical approach, duration of follow-up, timing to define failure, immediate outcomes of surgery including surgical margin, etc. [5-8].

This study demonstrated $87.8 \%$ of cure rate in primary resection which was in the range of $73 \%$ to $99 \%$ reported from previous studies $[5-8,11]$. On the other hand, our $12.2 \%$ failure rate was much lower than $26 \%$ reported by Serati et al which used CKC [5]. Their higher failure rate may lie on their longer follow-up period of 2 years in defining persistence/recurrence. On the other hand, Kanayama et al found low rates of persistent/recurrent diseases after laser conization: only $3.2 \%$

Table 4. Risk of Persistent/Recurrent CIN According to Clinical and/or Histopathologic Features $(N=385)$

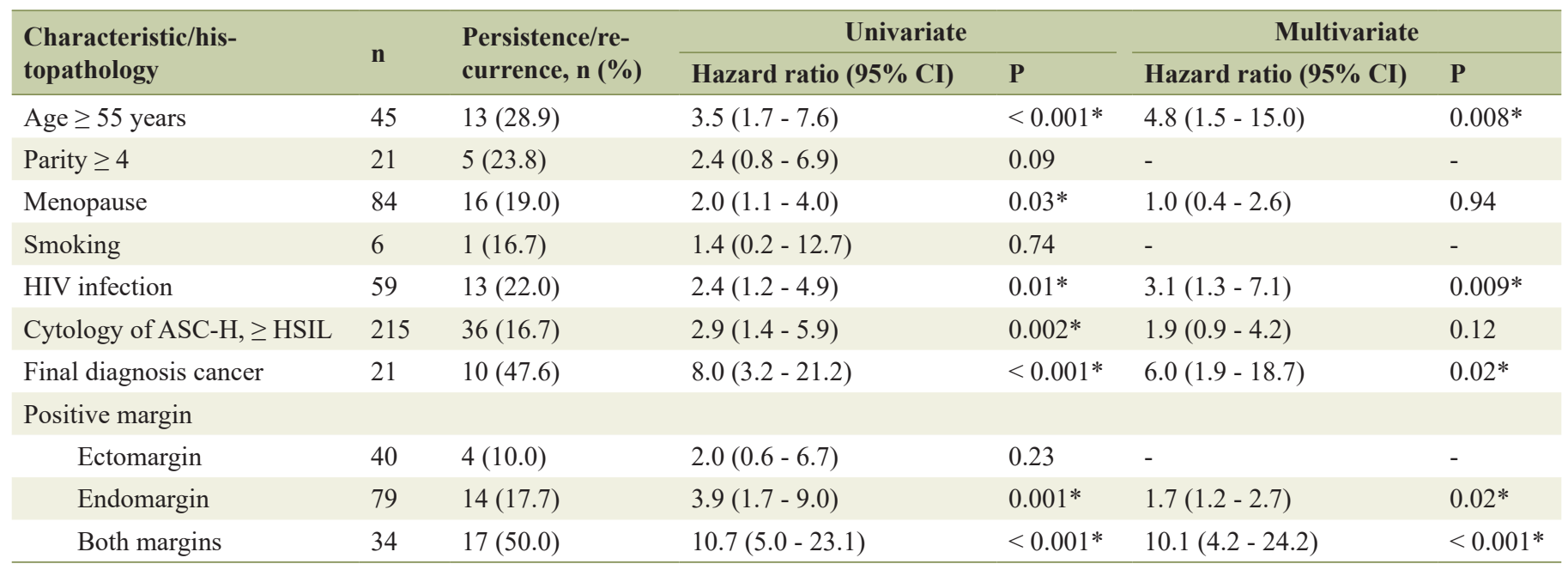

CIN: cervical intraepithelial neoplasia; HIV: human immunodeficiency virus; ASC-H: atypical squamous cells cannot exclude HSIL; HSIL: high-grade squamous cell intraepithelial lesion; CIS: carcinoma in situ; MIC: microinvasive carcinoma; Cl: confidence interval. 

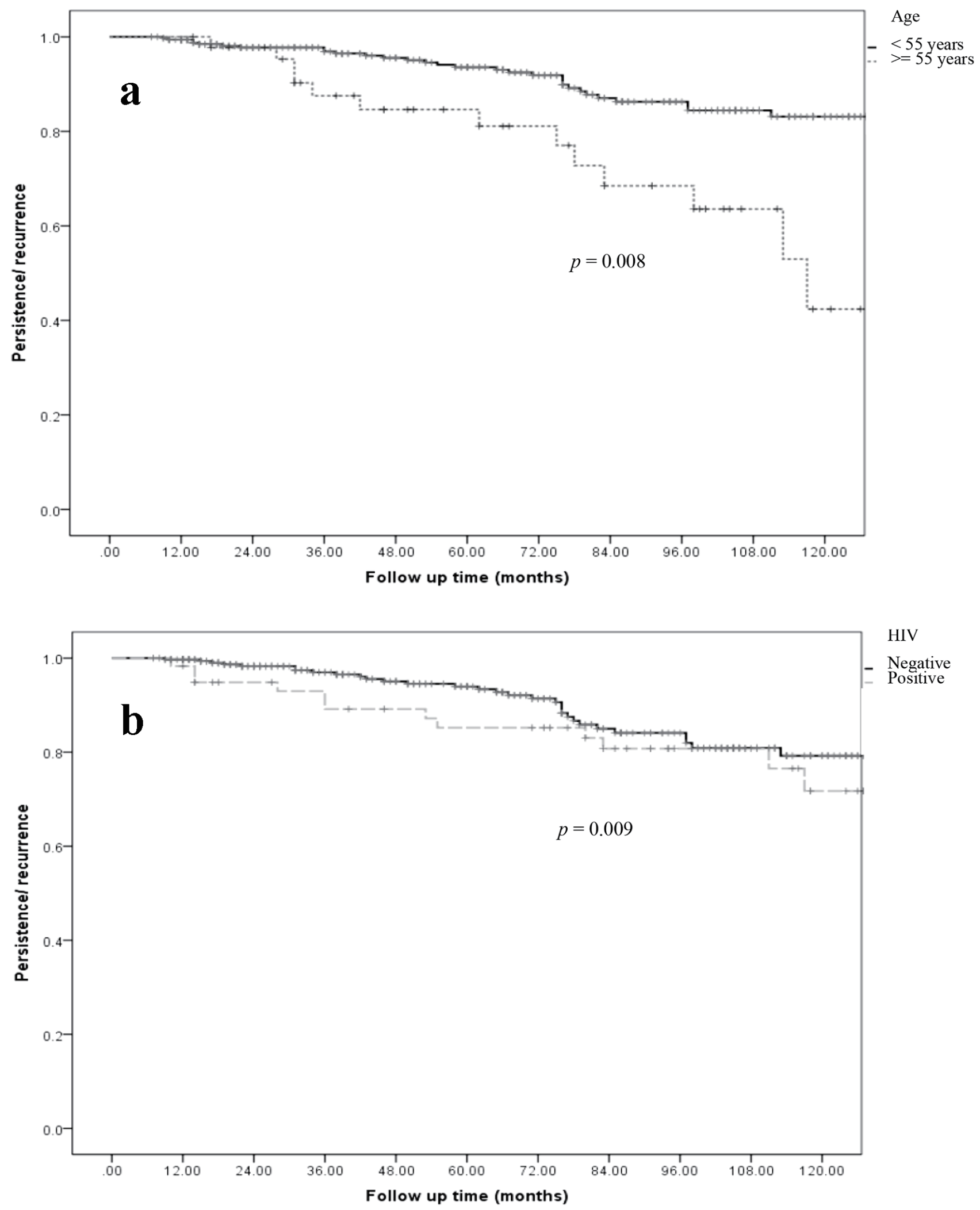

Figure 1. Progression-free survival (persistent or recurrent diseases) and clinical-pathological features. (a) Age group. (b) HIV infection status. (c) Final diagnosis of preinvasive or invasive cervical cancer. (d) Margin status after first LEEP. HIV: human immunodeficiency virus; LEEP: loop electrosurgical excision procedure. 


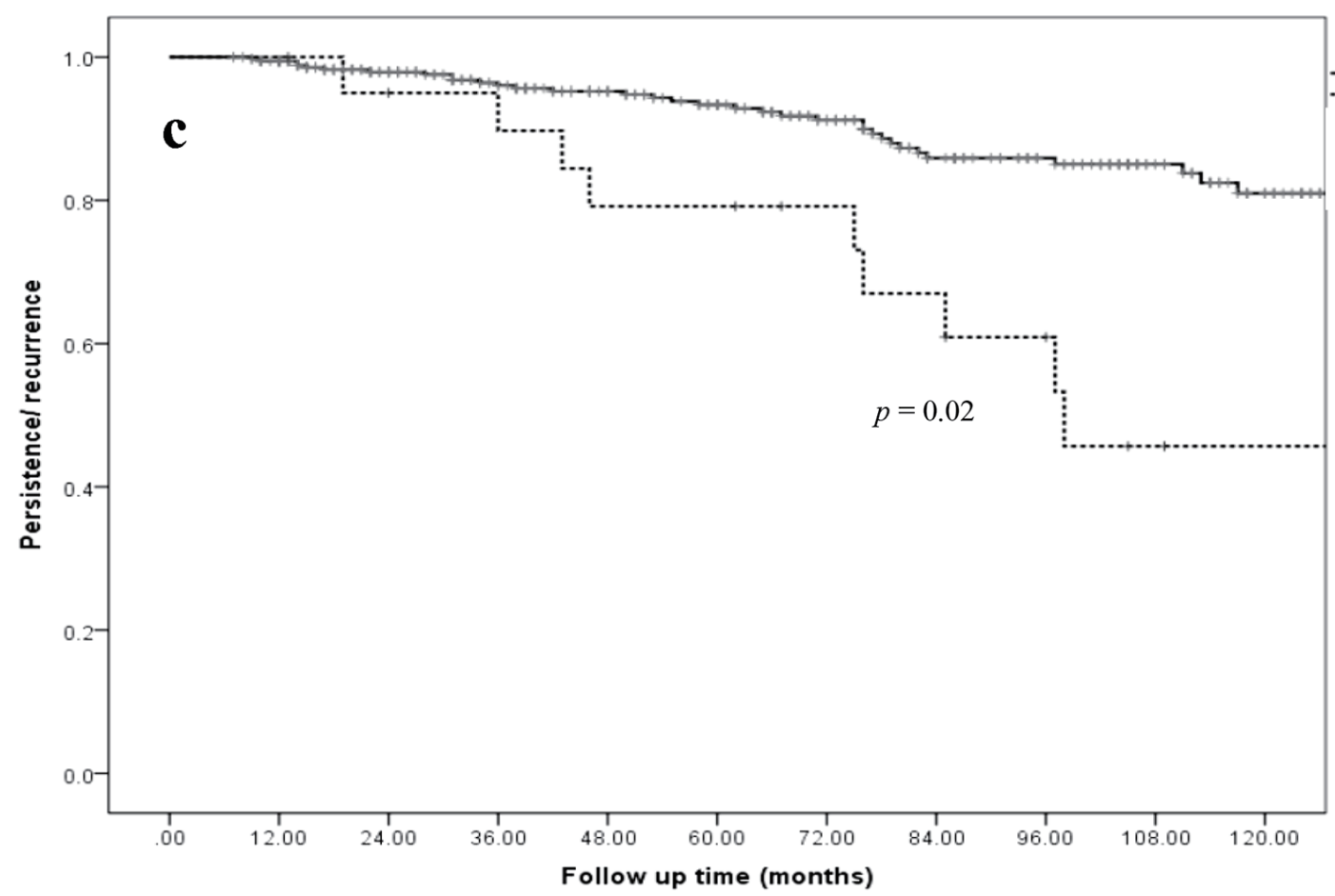

Final diagnosis

Preinvasive

Invasive cancer

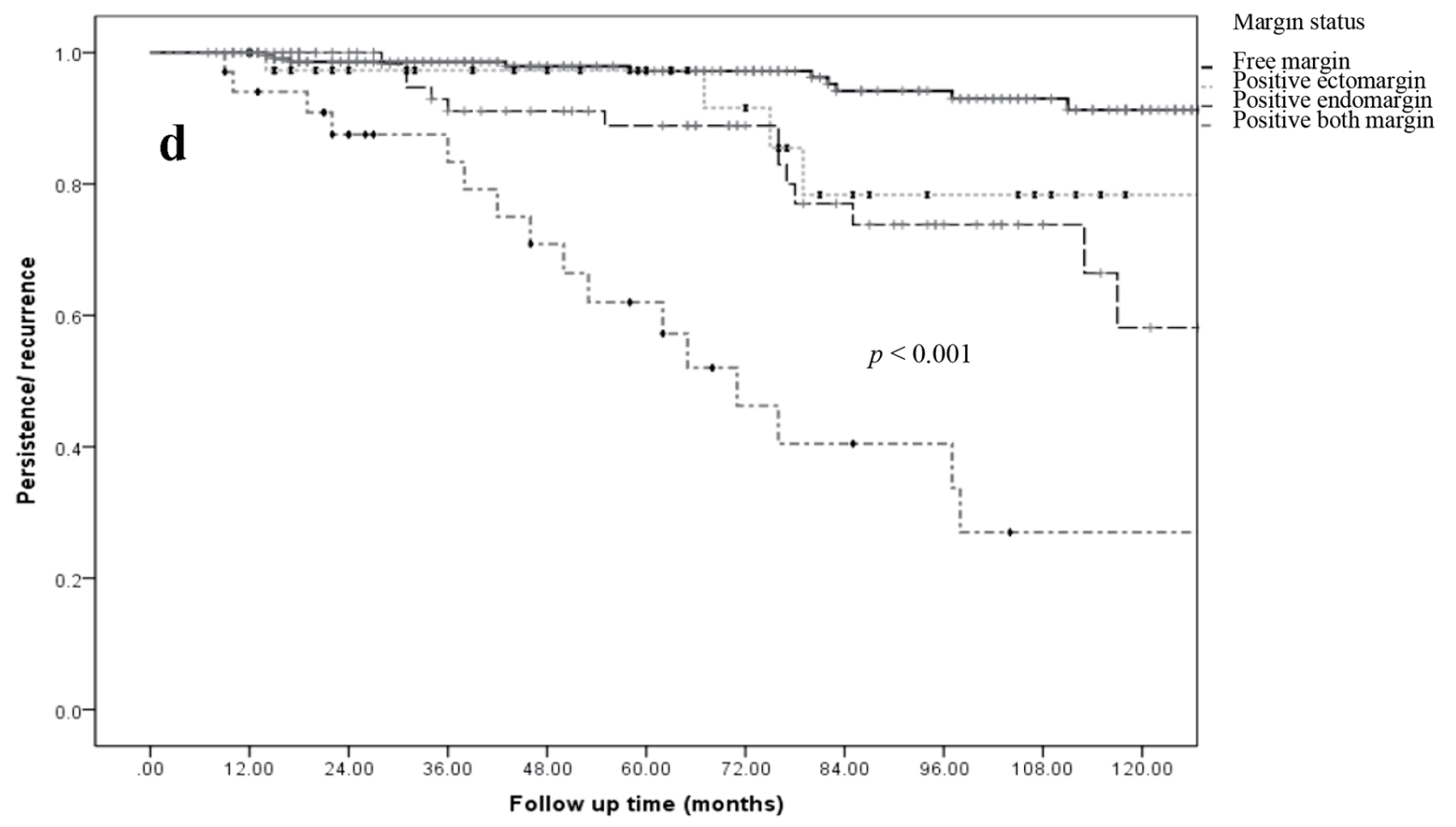

Figure 1. (continued) Progression-free survival (persistent or recurrent diseases) and clinical-pathological features. (a) Age group. (b) HIV infection status. (c) Final diagnosis of preinvasive or invasive cervical cancer. (d) Margin status after first LEEP. HIV: human immunodeficiency virus; LEEP: loop electrosurgical excision procedure.

for CIN 3 and $13.6 \%$ in MIC [6]. Their low failure rate may be due to a different technique (laser conization) as reflected by lower rates of positive margin than other studies: $8.9 \%$ in their study compared to $21.6 \%$ in Serati et al and $39.7 \%$ in our study.

For pre-operative features, the significant factors associ- 
ated with persistence/recurrence in our study were age older than 55 years and HIV infection. Our findings were consistent with previous studies which showed old age [12] or age more than 40 or 50 years $[13,14]$ were predictive factors of persistent or recurrent CIN. For HIV infection, previous studies also reported HIV infection as a significant factor for persistent/ recurrent diseases $[15,16]$. The other pre-operative predictors that had significant influence with persistent/recurrent CIN by univariate analysis in our study were parity of 4 or greater and cervical cytology report of ASC-H, HSIL, CIS, and carcinoma. Higher parity was also reported as a factor associated with recurrent diseases in previous studies (parity $\geq 4$ in Silva study and multiparity in Babkina study) $[14,16]$. However, these two factors were not confirmed as independent risk factors by multivariate analyses in our study.

For post-operative results, margin status was the only significant factor for recurrence or persistence. The clinical significance of positive resected margins was also observed in other studies. High incidences of persisted CIN ranging from $17 \%$ to $20 \%$ were found in patients with positive margin but would drop to as low as $2 \%$ to $6 \%$ in patients with complete resected margin $[11,17]$. One meta-analysis reported six-fold relative risk (RR) of persist/recurrent $\mathrm{CIN}$ after conization among patients with positive margin compared to those with complete excision [18]. The site of positive LEEP margin also has different impacts: positive endocervical margin was more clinically important than the ectocervical margin [11]. Persistence and recurrence were encountered similarly between those with positive endomargin or positive both endocervical and ectocervical margins, which were higher than those with negative margin or those with only positive ectomargin. For final diagnosis of cancer, our study demonstrated significance of persistence/recurrence in microinvasive and invasive cancer patients. These resembled with result of Kanayama et al's study [6], who concluded that residual/recurrent disease increased with the extent of disease (no patients with CIN 2, $3.2 \%$ with CIN 3 and $13.6 \%$ with MIC developed residual or recurrent disease). They also found significant residual of CIN and cancer in negative margin of conization (four of 38 patients, $10.5 \%$ ). These finding corresponded with our study that showed three of seven negative margin patients $(42.8 \%)$ had residual CIN 3 or CIS in hysterectomy specimens.

In summary, rate of failure after LEEP in our study was low. Independent significant factors associated with failure (persistence or recurrence) were age $\geq 55$ years, HIV infection, final diagnosis of cancer, and positive endomargin with or without ectomargin.

\section{Conclusion}

LEEP is an effective procedure of conization performed on patients with precancerous cervical lesions and early-stage cervical cancer. Our study demonstrated few events of minor complications and low rate of persistence/recurrence after LEEP. Intervention of re-excision or close follow-up is highly recommended for patients who had age $\geq 55$ years, HIV infection, final diagnosis of microinvasive or invasive cancer, or positive endocervical with or without ectocervical margin.

\section{Acknowledgments}

None to declare.

\section{Financial Disclosure}

The study was granted and supported from Navamindradhiraj University Research fund.

\section{Conflict of Interest}

None to declare.

\section{Informed Consent}

Not applicable.

\section{Author Contributions}

Jakkapan Khunnarong worked on protocol development, data collection, data cleaning, data analysis, and manuscript revision. Nitinan Bunyasontikul worked on protocol development, ethical approval, data collection, and drafting the manuscript. Siriwan Tangjitgamol worked on data analysis and manuscript revision.

\section{Data Availability}

The authors declare that data supporting the findings of this study are available within the article.

\section{Abbreviations}

AIS: adenocarcinoma in situ; AGC: atypical glandular cells; ASC-US: atypical squamous cells of undetermined significance; ASC-H: atypical squamous cells cannot exclude HSIL; CIN: cervical intraepithelial neoplasia; CIS: carcinoma in situ; CKC: cold knife conization; ECC: endocervical curettage; HIV: human immunodeficiency virus; HSIL: high-grade squamous cell intraepithelial lesion; LEEP: loop electrosurgical excision procedure; LSIL: low-grade squamous cell intraepithelial lesion; NILM: negative for intraepithelial lesion or malignancy; SCC: squamous cell carcinoma

\section{References}

1. Bray F, Ferlay J, Soerjomataram I, Siegel RL, Torre LA, Jemal A. Global cancer statistics 2018: GLOBOCAN estimates of incidence and mortality worldwide for 36 cancers in 185 countries. CA Cancer J Clin. 2018;68(6):394424. 
2. McCredie MR, Sharples KJ, Paul C, Baranyai J, Medley G, Jones RW, Skegg DC. Natural history of cervical neoplasia and risk of invasive cancer in women with cervical intraepithelial neoplasia 3: a retrospective cohort study. Lancet Oncol. 2008;9(5):425-434.

3. Cartier R, Sopena B, Cartier I. Use of the diathermy loop in the diagnosis and treatment of lesions of the uterine cervix. 4th World Congress of Cervical Pathology and Colposcopy. 1981. London.

4. Duggan BD, Felix JC, Muderspach LI, Gebhardt JA, Groshen S, Morrow CP, Roman LD. Cold-knife conization versus conization by the loop electrosurgical excision procedure: a randomized, prospective study. Am J Obstet Gynecol. 1999;180(2 Pt 1):276-282.

5. Serati M, Siesto G, Carollo S, Formenti G, Riva C, Cromi A, Ghezzi F. Risk factors for cervical intraepithelial neoplasia recurrence after conization: a 10-year study. Eur J Obstet Gynecol Reprod Biol. 2012;165(1):86-90.

6. Kanayama S, Nakagawa E, Ueno S, Muraji M, Wakahashi S, Sudo T, Yamada T, et al. Outcomes of laser conization for cervical intraepithelial neoplasia 2-3 and microinvasive cervical cancer. World J Oncol. 2014;5(2):62-67.

7. Simoes RB, Campaner AB. Post-cervical conization outcomes in patients with high-grade intraepithelial lesions. APMIS. 2013;121(12):1153-1161.

8. Leguevaque P, Motton S, Decharme A, Soule-Tholy M, Escourrou G, Hoff J. Predictors of recurrence in high-grade cervical lesions and a plan of management. Eur J Surg Oncol. 2010;36(11):1073-1079.

9. Saslow D, Runowicz CD, Solomon D, Moscicki AB, Smith RA, Eyre HJ, Cohen C, et al. American Cancer Society guideline for the early detection of cervical neoplasia and cancer. CA Cancer J Clin. 2002;52(6):342-362.

10. ACOG committee opinion no. 300. Cervical Cancer Screening in Adolescents. American college of obstetrics and gynecology. Obstet Gynecol. 2004;104:885-889.
11. Gardeil F, Barry-Walsh C, Prendiville W, Clinch J, Turner MJ. Persistent intraepithelial neoplasia after excision for cervical intraepithelial neoplasia grade III. Obstet Gynecol. 1997;89(3):419-422.

12. Verguts J, Bronselaer B, Donders G, Arbyn M, Van Eldere J, Drijkoningen M, Poppe W. Prediction of recurrence after treatment for high-grade cervical intraepithelial neoplasia: the role of human papillomavirus testing and age at conisation. BJOG. 2006;113(11):1303-1307.

13. Lu CH, Liu FS, Kuo CJ, Chang CC, Ho ES. Prediction of persistence or recurrence after conization for cervical intraepithelial neoplasia III. Obstet Gynecol. 2006;107(4):830-835.

14. de Mello Silva MV, Coutinho IC, de Andrade Heraclio S, Fittipaldi HM, Jr., Katz L. Factors associated with the persistence/recurrence of CIN2/3 in women submitted to loop electrosurgical excision procedure in a teaching hospital in northeastern Brazil: a case-control study. J Low Genit Tract Dis. 2014;18(4):286-290.

15. Malapati R, Chaparala S, Cejtin HE. Factors influencing persistence or recurrence of cervical intraepithelial neoplasia after loop electrosurgical excision procedure. J Low Genit Tract Dis. 2011;15(3):177-179.

16. Babkina N, Heller DS, Goldsmith LT, Houck KL. Cervical conization for cervical intraepithelial neoplasia (CIN) 2 and 3 in HIV-positive women: a case-control study. J Low Genit Tract Dis. 2015;19(2):110-114.

17. Livasy CA, Maygarden SJ, Rajaratnam CT, Novotny DB. Predictors of recurrent dysplasia after a cervical loop electrocautery excision procedure for CIN-3: a study of margin, endocervical gland, and quadrant involvement. Mod Pathol. 1999;12(3):233-238.

18. Ghaem-Maghami S, Sagi S, Majeed G, Soutter WP. Incomplete excision of cervical intraepithelial neoplasia and risk of treatment failure: a meta-analysis. Lancet Oncol. 2007;8(11):985-993. 